\title{
STRATEGY OPTIMIZATION AND GENERATION FOR CONSTRUCTION PROJECT MANAGEMENT USING AN INTERACTIVE SIMULATION
}

\author{
Pei Tang \\ Amlan Mukherjee \\ Department of Civil and Environmental Engineering \\ Michigan Tech. \\ Houghton, MI 49931, U.S.A. \\ Department of Civil and Environmental Engineering \\ Michigan Tech. \\ Houghton, MI 49931, U.S.A.
}

\author{
Nilufer Onder \\ Department of Computer Science \\ Michigan Tech. \\ Houghton, MI 49931, U.S.A.
}

\begin{abstract}
Construction activities are exposed to unpredictable external events and internal dynamic feedbacks of constraints which deviate projects from as-planned duration and costs. In practice, various decisions have been used to minimize the impacts of risks. Learning from experiences is valuable which requires historical data collection and analysis. To avoid the high cost in direct data collection and difficulties in studying single decision impacts, we present an alternative to study and optimize decision strategies. Interactive Construction Decision Making Aid (ICDMA) is an interactive simulator which allows users to implement different decision strategies on the defined projects. All the project information and decision data in the simulation are recorded electronically. We started with five candidate strategies and analyzed data for general patterns. New hybrid strategies were generated based on the data analysis. Reimplementation of new strategies showed improvement in cost and duration management, validating the feasibility of strategy optimization through interactive simulation.
\end{abstract}

\section{INTRODUCTION}

Construction project management involves managing complex situations characterized by unpredictable external events, and internal dynamic feedbacks that cause the project to deviate from as-planned duration and cost. Construction managers make decisions to control the budget and duration of projects. Historical data analysis are useful for examining and learning from experiences. However, a rigorous collection of decision-making data during the construction process can prove to be practically difficult and expensive. Besides, case studies do not allow analysis of what-if scenarios to compare the effects of combined decisions and different decision sequences. In this paper, we propose to study alternative decision-making strategies using an interactive simulated environment that allows the exploration of multiple deviations from the as-planned project progress in cost and duration management.

A construction project's final outcome is highly dependent on the dynamic interactions between resources on site - as directly controlled by management decisions. Each decision sets the project on an unique trajectory through a space of future possibilities. For example, a decision to crash a specific activity using over-time work may cause the crews to intersect the path of other spatially co-located activities and increase congestion on site. This may not have the desired impact on reducing the duration of the project. Therefore, an examination of the effectiveness of a management decision requires consideration of its immediate expected impact, as well as subsequent situations and future decisions that result from it. Decision-making can not be studied as an isolated act, but rather as a dynamic process where each successive decision is made in consideration of previous decision outcomes and possible future scenarios. This requires the study and comparison of alternative decision-making strategies. 
A decision-making strategy provides a coherent framework for considering decision sequences that consider unexpected future outcomes and controls the budget and schedule performance. It is defined as a guideline and direction that provides the basis for a family of acceptable decisions towards achieving a desired project outcome.

In this paper, we used an interactive simulation to examine some candidate strategies, and then generate new hybrid strategies through an iterative optimization process. Specifically, a construction project scenario was first simulated in the construction platform using cost and schedule data from a real life construction project that had been verified to run correctly with the simulation platform. The scenario was designed at a mid-point in the project, where it has already incurred a schedule delay and cost overrun. Five candidate strategies were created and implemented within this scenario to manage the crisis situation over a window of seven time advances. The project status and decision data in the simulation for each strategy were collected and analyzed. The advantageous management properties on those candidate strategies were investigated with the proposed analysis method. The findings in advantageous properties were used to generate new hybrid strategies that were expected to have better performance. The performance of the new hybrid strategies can be further analyzed iteratively until the best decision-making strategy is produced.

The fundamental contribution of this research is in the iterative interactive simulation method it provided in optimizing hybrid strategies during the planning phase of construction project management. This method can be applied across diverse construction projects using the simulation platform described in this paper. Given the non-prototypical nature of construction projects and the strong dependency on site specific context, we believe a standard method that can provide project specific solutions is an important contribution. When applied over multiple projects, it may point to significant effective decision-making patterns that can be generalized across all construction scenarios. The absence of any such general patterns will in no way diminish the value of this general method and its applicability in improving construction management planning.

\section{BACKGROUND}

Computerized methods have been used for decades in studying construction decision making with the advantages of providing different simulation scenarios, automation, and electronic data collection. Kaklauskas et al. (2007) developed an on line multiple criteria decision support system for construction projects that considered the qualitative, technical, technological and other characteristics for construction alternatives. A group decision-making framework with linguistic preference relations was used for distributed decision-making (Herrera-Viedma et al. 2005). It used a guidance advice system as a feedback mechanism to automate the decision-making process. Yates (1993) introduced a delay analysis system (DAS), which had the ability to determine possible causes for project delays and suggested alternative actions to prevent further delays by allocating human and material resources. Analytical hierarchy process was also used to aid decision-making in supplier selection (Ghodsypour and O'Brien 1998) using linear programming.

A type of interactive computerized method, characterized by the interactive communication between a simulator and decision makers, has been used. The computerized simulator updates the project situation by taking in the decisions. Decision makers respond to the consequences resulting from previous decisions. Such interactive simulations have already been used to aid real-time optimization of water resource system operations, to develop training programs for system managers and technicians, and to demonstrate system management alternatives (Cunningham and R. 1984, Cunningham and R. 1986). Makropoulos et al. (2003) established a fuzzy logic spatial decision support system in urban water management with the ability to explore predefined strategies and incorporate users' optimization preference to identify an optimal composite strategy. Kim and Choi (2001) developed an interactive group support system with the ability to identify conflicting opinions in the group and suggest new directions. Malakooti (1988) created a multiple criteria decision-support system, and used heuristic interactive approaches to optimize the utility function by allowing users to input their preferences.

Based on the advantages of applying interactive computerized methods to study decision making in different areas, we presented a method to optimize and generate decision making strategies in construction management by iteratively implementing strategies and analyzing the data obtained from interactive simulation runs.

\section{INTERACTIVE SIMULATION AND METHODOLOGY}

Interactive Construction Decision Making Aid(ICDMA) is a specific implementation of a generalpurpose interactive simulation framework (Rojas and Mukherjee 2006, Watkins et al. 2008). It sim- 
ulates a construction project based on the as-planned schedule and costs. The user of the simulation takes on the role of construction manager whose goal is to complete the project on schedule and under budget. During the simulation run, the user is presented with random external events that force the simulated project to deviate from its original plan. The user has to respond to those events by making decisions on resource allocation. The consequences from the decisions result in new scenarios for the user to respond to. This process continues until the completion of the simulated construction project.

The underlying models in ICDMA are capable of simulating the as-built performance of the actual project (Anderson et al. 2009). The querying algorithm in ICDMA is capable of forecasting the likelihood of future scenarios and the likely project outcomes from any point in the project using current project information by querying a space of simulated project futures. A space of possible project outcomes consisting of future traces under varying conditions, constraints and decision are generated and analyzed using a Monte Carlo sampling method. Each candidate in the future space is a complete simulation trace of the project. In this research, the querying number is set as 1,000, which means that 1,000 projected total costs and durations are predicted at the end of each simulation step. The actual project outcome is the most likely scenario in the space of possible outcomes and can be described by the distribution of 1,000 projected results.

We assume that there exist patterns in efficient strategy groups and that investigation into patterns could help reveal the mechanisms driving those strategies for a specific project, which contributes to the development and optimization of new strategies. In this paper, we generated new hybrid strategies by statistically analyzing and clustering the simulated future outcome space of alternative decision strategies that were collected from the interactive simulation platform. A potentially problematic scenario was established and five different control strategies to manage the crisis situation were explored in an interactive simulation mode. Hierarchical cluster analysis was used to cluster strategies and results were concluded, based on which hybrid strategies were generated. The newly generated hybrid strategies were implemented in the same project scenario for comparison. The methodology was shown in Figure 1.

\section{EXPERIMENT}

\subsection{Project Description}

ICDMA implements the construction of a four-storey structural steel framed office building that was built in real life. The simulation was based on the schedule and construction information documented by (Daccarett and Mrozowski 1999). It was a structural steel framed office building built in 2003, consisting of four stories, 80,000 square feet of built area, and approximately 400 tons of structural steel. During the construction planning stages, the steel frame building was divided into six sequences, each of which involved three activities: hoisting, bolting and decking.

In order for the crews to be efficiently utilized, it was important to minimize crew idle time. The project was planned in such a way that certain project activities could be implemented in parallel to improve the efficiency of the construction process. For instance, after the bolting crews finished fastening the first sequence of steel, they could immediately begin the second sequence while the decking crews started to lay the steel decking across the steel members of the first sequence. Three types of crews were used in this simulation: crane crew, steel crew and welder crew. All related information was input into the database through a web based interface. The discussion of the project set-up is not a part of the scope of this paper and can be found in (Anderson et al. 2009). A snapshot of the project and events setup is in the left column of Figure 1.

\subsection{Project Scenario and Strategy Description}

After creation of the project, the probabilities of external events that were expected to occur in the project were set in the database. For illustration purposes, the external events were assumed to be rain (major weather), snow (extreme weather), labor strike, material delivery failure and worker sickness. Their respective probabilities were set at $8 \%, 2 \%, 1 \%, 6 \%$, and $10 \%$. Hence, the probability it will rain is $8 \%$ each time the simulator is advanced. In a realistic application, such probabilities can be estimated from site specific records. The space capacity was set to be 3,000 units. If the space occupied by the material exceeded 3,000 units, a space conflict event occurred. Without the uncertain events, the simulated project will be completed on time with as-planned cost. These probabilities are specific to the project at hand, and the investigators using the simulation can choose appropriate values to reflect the circumstances of their projects. After the setup of event probabilities, the project was simulated for fourteen weeks on the simulation platform. At the end of the fourteenth week, 
Tang, Mukherjee and Onder

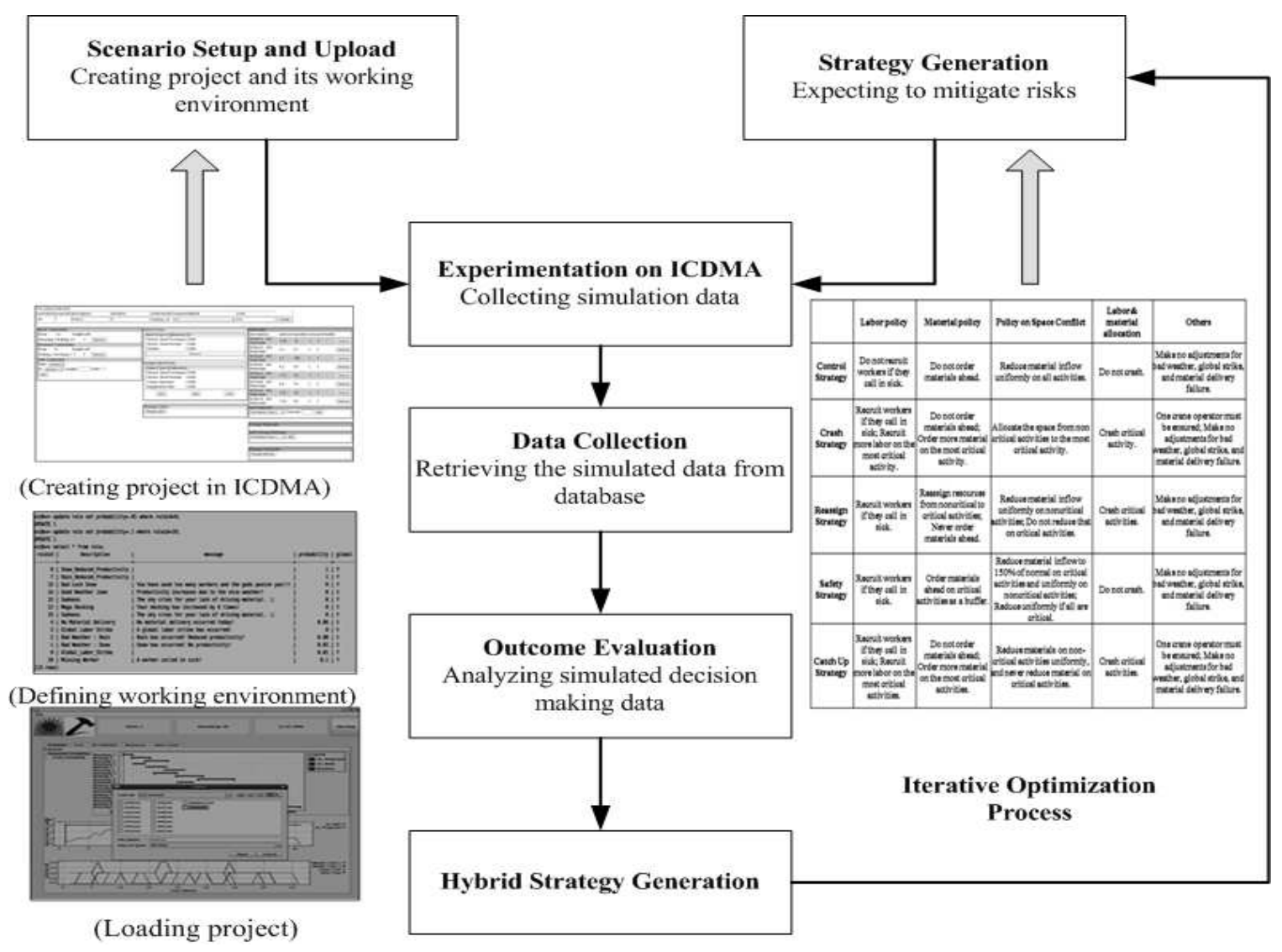

Figure 1: Methodology of hybrid strategy generation

the project was found to be delayed, requiring another 39 weeks to complete the project with a total cost of $\$ 1,863,589$. This meant that the project was predicted to be exceeding the as-planned cost by $25 \%$ and as-planned duration by $30 \%$ at the completion. The decision-making problem at hand was to develop a strategy that can manage the crisis situation at that point.

After the scenario was set up, five candidate decision strategies were established. A control strategy was used to reflect baseline conditions, and the other four strategies were chosen as alternative approaches to managing the project at hand. In this case, the Control Strategy aimed at completing the project by closely adhering to a baseline decision sequence. The Crash Strategy tried to crash the schedule actively. The Reassign Strategy aimed to optimize the project by prioritizing the activities on the critical path. The Safety Strategy aimed at reducing the risks due to delayed material delivery by ordering materials ahead of time. The CatchUp Strategy tried to catch up with the schedule passively when falling behind. Theoretically, decision makers using this method could start with any set of initial strategies and iteratively correct them till the best strategy is identified. Starting with a list of suitably chosen strategies can reduce the number of iterations. Creating a strategy involves defining the guidelines in ordering and allocating material, labor and equipment resources under different uncertain situations. Techniques for defining strategies are beyond the discussion of this work.

To examine the performance of each strategy, each strategy was implemented interactively in a critical scenario for a period of seven simulation weeks from week 15 to week 21 . During each simulation week, the decision maker managed the project by allocating resource and adhering to the guidelines of strategies under investigation. The decision maker not only made decisions at the beginning of each simulation week based on a judgment of the situation at hand, he/she also had to manage risky random external events in the middle of simulation. Each strategy was implemented for thirty five runs to meet the minimum requirement of statistical analysis. For the sake of uniformity and given the experimental nature of this research, a single decision maker ran all the simulations. 
Tang, Mukherjee and Onder

\begin{tabular}{ll}
\hline Events & Impacts \\
\hline Global labor strike & $\begin{array}{l}\text { In the case of a global labor strike, there } \\
\text { would be no productivity. Material supply is } \\
\text { not impacted. }\end{array}$ \\
\hline Rain & $\begin{array}{l}\text { Rain has negative impacts on productivity, if } \\
\text { serious, results in no productivity. The severity } \\
\text { is decided when the event occurs. Materials } \\
\text { are supplied as scheduled. }\end{array}$ \\
\hline Snow & $\begin{array}{l}\text { Snow has negative impacts on productivity, if } \\
\text { serious, results in no productivity. The severity } \\
\text { is decided when the event occurs. Materials } \\
\text { are supplied as scheduled. }\end{array}$ \\
\hline Material delivery failure & $\begin{array}{l}\text { In the case of a material delivery failure, ma- } \\
\text { terials in stock would be used first. No work } \\
\text { would be done when there are no materials } \\
\text { available. }\end{array}$ \\
\hline
\end{tabular}

Table 1: Impacts of global events that might occur in the simulation

The simulation performance data at each decision control point was collected and stored for further analysis.

\section{DATA ANALYSIS AND HYBRID STRATEGY GENERATION}

\subsection{Preliminary Analysis}

As explained in section 3, at the end of each simulation step 1,000 query results were produced, from which the average projected duration and cost of the project were calculated. The cost and duration exceedance were calculated as a percentage by comparing the projected results with the as-planned cost and duration. The equation used was:

ExceedancePercentage $=\frac{\text { ProjectedTotalCost }(\text { Duration })-\text { AsplannedTotalCost }(\text { Duration })}{\text { AsplannedTotalCost }(\text { Duration })} * 100 \%$.

The exceedance percentages were used as a metric to study each strategy's response to a specific project scenario across 7 weeks. A stacked column histogram was created from 35 sets of projected cost or duration exceedance percentages.

In the stacked histograms, the horizontal axis represented different strategies and sub-horizontal axis represented the seven simulation weeks. The vertical rectangles represented a frequency distribution of the 35 runs, classified across cost and duration exceedence categories. Seven categories of cost exceedance percentage were set to $10 \%, 12 \%, 14 \%, 16 \%, 18 \%, 20 \%$ and $22 \%$. When a run is classified in the category of $12 \%$, it means that the final project cost for the specific run is $12 \%$ beyond the budget. When a run has a cost exceedence between $12 \%$ and $14 \%$, it is classified in the category of $14 \%$. The projected duration exceedance percentages were analyzed using the following six categories: $16 \%, 20 \%, 24 \%, 28 \%, 32 \%$ and 36\%. For example, in Figure 2, for the Catch-Up Strategy in week 15, all the 35 runs had a projected cost exceedance percentage in the range of $16 \%$. In week 21, projected cost exceedance percentages of 13 runs were in the range of 10\%, 14 runs in the range of $12 \%, 6$ runs in the range of 14\%, 1 run in the range of $16 \%$ and one in the range of $18 \%$. It should be noted that the lower the vertical sub-rectangle was in the frequency distribution, the smaller the exceedence percentages it represented.

In Figure 2, the exceedance percentage of almost all the runs' were in the range of 16\%, shown by white vertical rectangles in week 15 and week 16. This was an indication that strategies were equally efficient in cost control in the first two weeks. Starting from week 17, except the Reassign Strategy, all strategies had more runs with lower cost exceedance percentages out of 35 runs. This can be found by comparing the dark areas under the white vertical rectangles. This was a sign that all the strategies except the Reassign Strategy had more or less reduced project costs. In Figure 3, about $90 \%$ of the projected duration exceedance percentages were in the range of $32 \%$ at the end of the first two weeks for all the strategies. There were no improvements in the first two weeks compared to the $30 \%$ duration exceedance in week 14. In the following five weeks, the CatchUp Strategy, the Crash Strategy and the Reassign Strategy all showed decreases.

Except the expected general trend towards lower cost across seven weeks, strategies exhibited different behaviors in managing project cost and duration. The predicted cost began to decrease since 


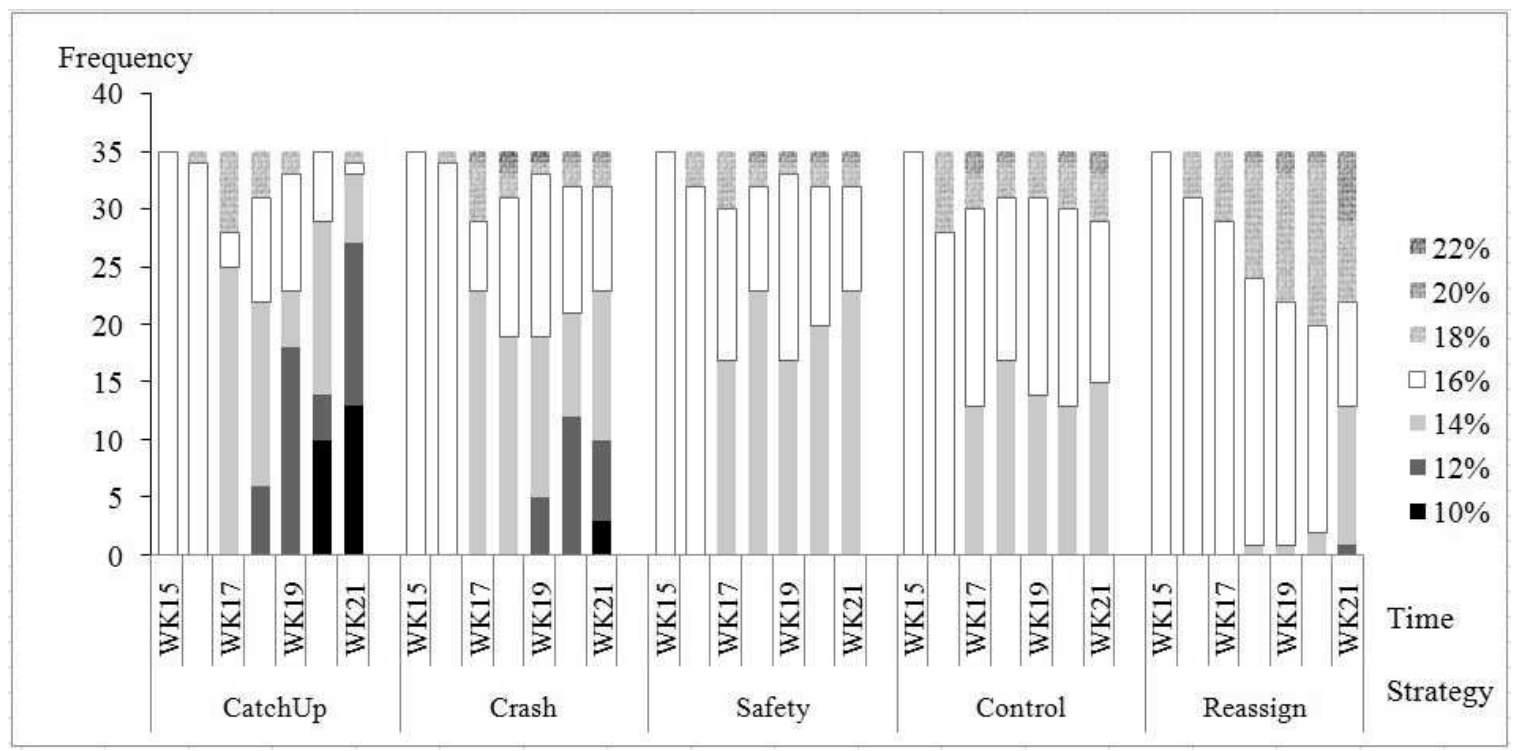

Figure 2: Stacked histogram of cost exceedance percentage across seven weeks under five strategies

week 17 on all the strategies except for the Reassign Strategy while the costs began to decline during week 21 under the Reassign Strategy. Furthermore, the CatchUp Strategy and Crash Strategy reduced more cost and duration at a faster speed than the Safety Strategy and the Control Strategy. The Safety Strategy and Control Strategy further delayed the completion time. However, the Safety Strategy and the Control Strategy had a smaller deviation compared to the CatchUp and the Crash Strategies, as illustrated by the relatively stable area under the white vertical rectangle for the Safety Strategy and the Control Strategy across seven weeks. The Reassign Strategy was good at duration management but was not cost efficient.

From the above description, the Control Strategy and the Safety Strategy maintained a low variation while the CatchUp strategy and Crash Strategy had a faster speed in mitigating project duration delay and cost overrun. The Reassign Strategy was efficient in mitigating project duration delay but might result in more serious cost problems. We hypothesize that those strategies with similar outcomes have similar mechanisms driving them. Strategies with similar outcomes are regarded as one group. Investigation of general patterns and comparison between different groups could help reveal the mechanism driving the strategies, thus contributing to the development and optimization of new strategies. However, difficulties existed in classifying the strategies based on a histogram of projected cost and duration exceedance percentages. Hierarchical cluster analysis, an exploratory data analysis tool aimed at sorting different objects into groups, was used as a solution to this problem in the next section.

\subsection{Hierarchical Cluster Analysis}

The variables in the cluster analysis were projected cost and duration exceedance percentages during each week under each specific strategy. The cases were labeled by strategies. A dendrogram was chosen to indicate the formation of coherent clusters. Wards method was used to create clusters because it minimizes the sum of squares of any pair of clusters to be formed at a given step. Z-score was chosen to standardize the data to eliminate the effects of the variance of variables.

The dendrogram in Figure 4(a) was created using projected cost exceedance percentages. The Safety Strategy and the Crash Strategies were clustered in one group. The Control Strategy was closer to the Safety and Crash Strategy group than CatchUp Strategy and the Reassign Strategy were. Thus, the first group included the Safety Strategy, the Crash Strategy, and the Control Strategy while the Catch-Up Strategy and the Reassign Strategy were in the second and third group. The Control Strategy and Safety Strategy were not in the same group because the Control Strategy was less efficient in reducing cost and duration than the Safety Strategy in Figure 5. The differences between the two decision strategies were that material was ordered ahead under the Safety Strategy. If materials were 


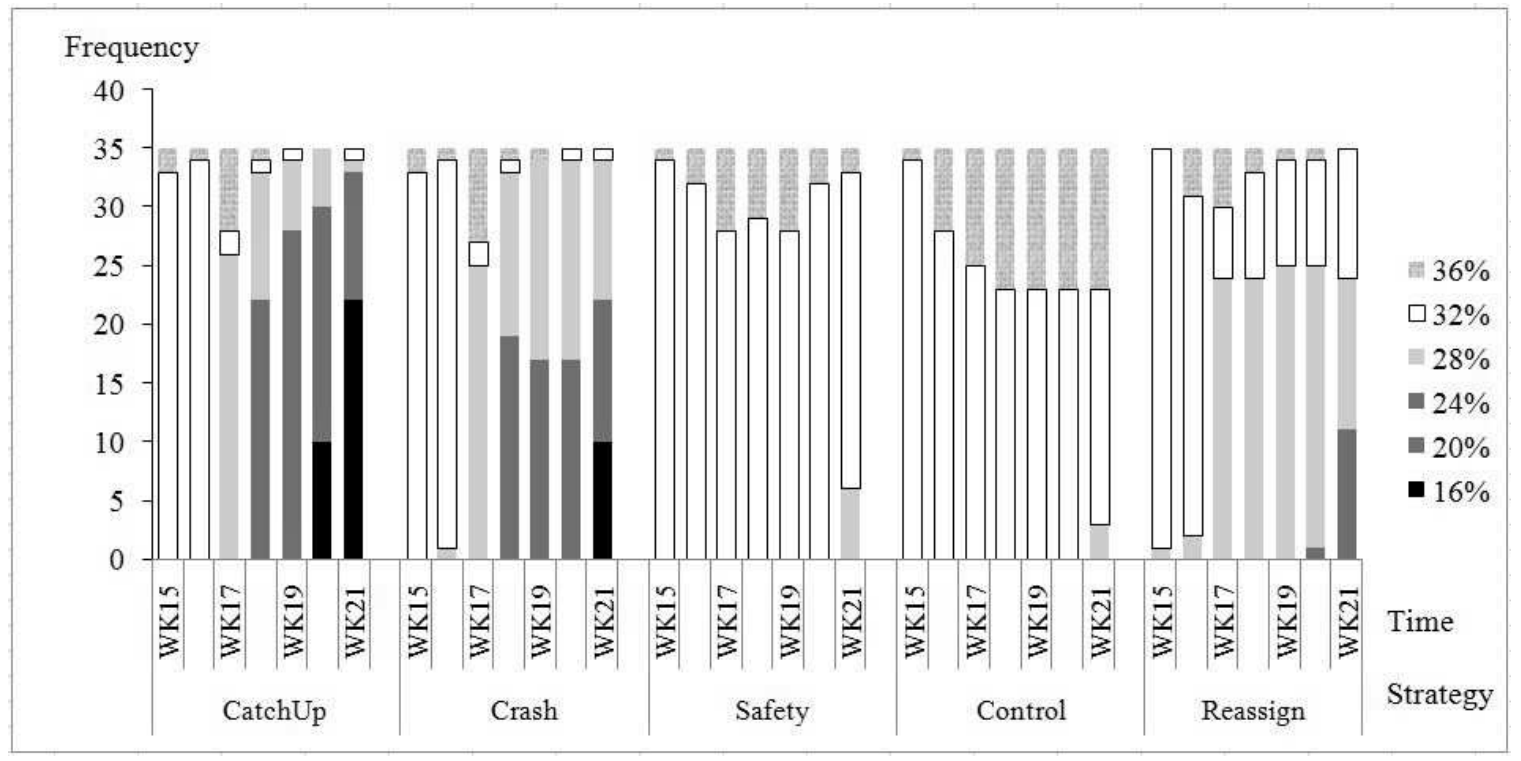

Figure 3: Stacked histogram of duration exceedance percentage across seven weeks under five strategies

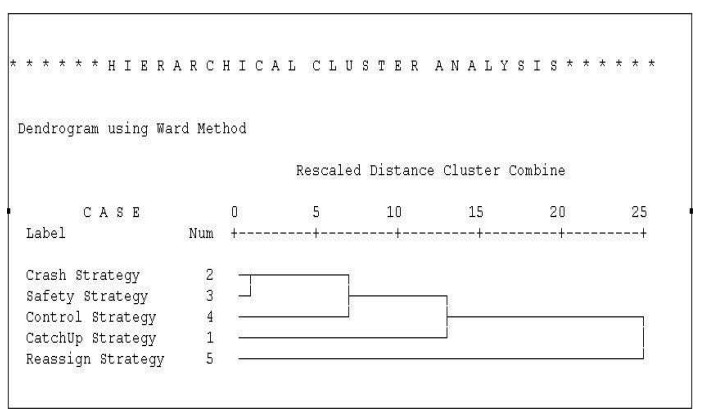

(a)

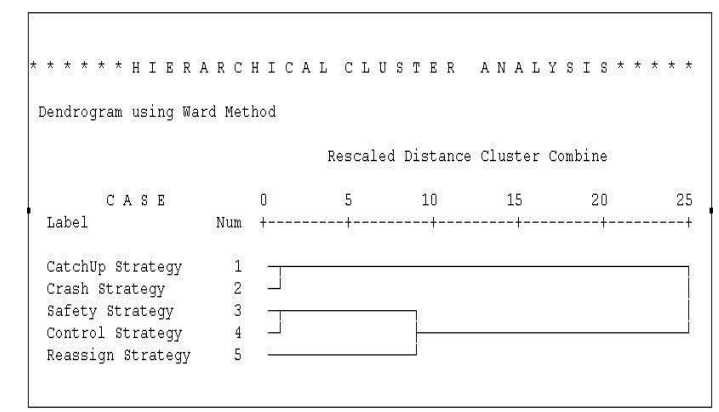

(b)

Figure 4: (a)Cluster result using cost exceedance percentage (b)Cluster result using duration exceedance percentage

out of stock, the project can be delayed in case of material delivery failure. Figure 6 illustrated that the project had lower predicted exceedance percentages of duration under the Safety Strategy than that under the Control Strategy. In Figures 5 and Figure 6, the predicted exceedance percentages of cost and duration under the CatchUp strategy were smaller than that under the Crash Strategy.

The dendrogram in Figure 4(b) showed that the strategies can be grouped into two clusters using projected duration exceedance percentages. The first cluster included the Control Strategy and the Safety Strategy, and the second cluster included the Crash Strategy, the CatchUp Strategy, and the Reassign Strategy. Comparisons between two clusters indicated that increasing labor and resource input on the critical activity reduced duration, though its impacts on cost may vary. Critical differences between the first strategy group and the second strategy group were that the later group had more labor and resource inputs on the critical activities. From Figures 6, the second group was more efficient in minimizing duration than the first group.

There were two sub-clusters within the second cluster. The first sub-cluster consisted of the Crash Strategy and the CatchUp Strategy, and the second sub-cluster consisted of the Reassign Strategy. The sub-clusters provided a more nuanced perspective on the differences between the strategies. Investigating two sub-clusters helped to explain whether a strategy was cost effective depended on the way non-critical activities were completed. The differences between the Crash Strategy and the CatchUp Strategy under the sub-cluster were that the Crash Strategy tried to be ahead of schedule actively by crashing critical activities while CatchUp strategy crashed activities 


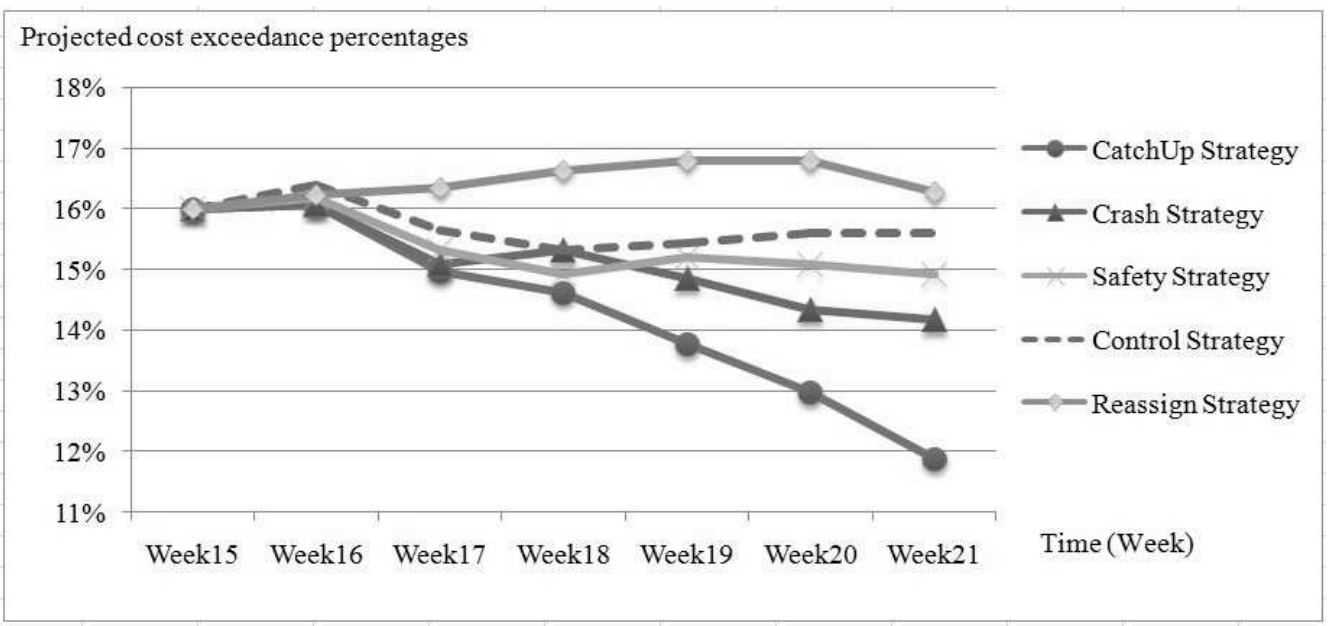

Figure 5: Management of cost exceedance under five strategies

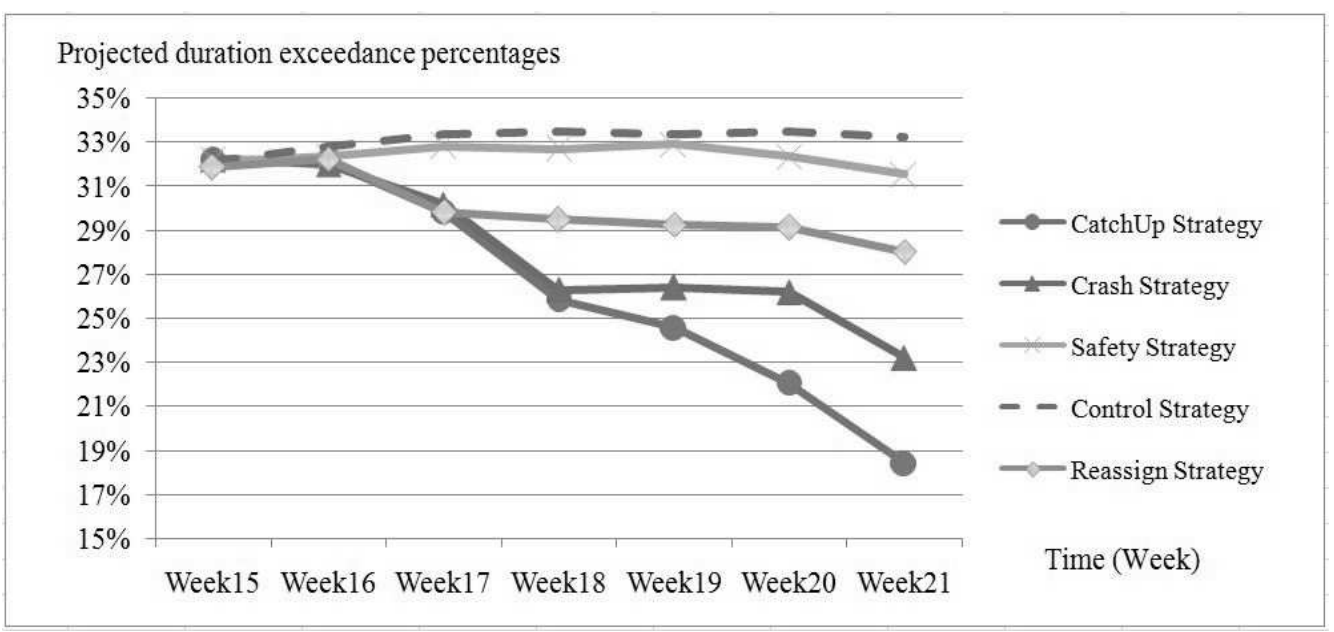

Figure 6: Management of duration exceedance under five strategies

including both noncritical activities and critical activities passively when falling behind. The CatchUp strategy performed better in controlling cost and duration than the Crash Strategy did, which indicated that increasing resource input into more than one activity seemed to be more efficient in minimizing cost and duration. The differences between the first sub-cluster, the Crash and CatchUp Strategy, and the second sub-cluster, the Reassign Strategy, in Figure 4(b) were that noncritical activities were impacted when resources were moved from noncritical activities to critical activities under the second sub-cluster, while noncritical activities were hardly impacted under the first sub-cluster.

These differences showed that increasing labor and resource inputs on critical activities was cost effective only if the importance of labor and material inputs on noncritical activities was recognized as well. When the labor and material inputs were moved from noncritical activities to critical activities, critical activities were crashed with increasing the duration of noncritical activities. Beyond some threshold, the durations of the non-critical activities increased to the point where they became critical, incurring more cost, especially when the cost to crash them was higher than the amount saved by crashing the critical activities. This explained the rising trend of cost exceedance under the Reassign Strategy in Figure 5. 
Tang, Mukherjee and Onder

\begin{tabular}{|c|c|c|c|c|}
\hline & $\begin{array}{l}\text { Labor Order } \\
\text { Policy }\end{array}$ & $\begin{array}{l}\text { Material Order } \\
\text { Policy }\end{array}$ & $\begin{array}{l}\text { Labor and material } \\
\text { allocation }\end{array}$ & $\begin{array}{l}\text { Policy on Space } \\
\text { Confliction }\end{array}$ \\
\hline $\begin{array}{l}\text { Hybrid Strategy } \\
1\end{array}$ & $\begin{array}{l}\text { Recruit workers if they } \\
\text { call in sick; }\end{array}$ & $\begin{array}{l}\text { Always order materials } \\
\text { ahead on critical activity } \\
\text { and order materials equally } \\
\text { on non critical activities if } \\
\text { possible. }\end{array}$ & $\begin{array}{l}\text { Crash the critical activ- } \\
\text { ities by allocating ex- } \\
\text { tra labor and material } \\
\text { to activities in case of } \\
\text { delay. }\end{array}$ & $\begin{array}{l}\text { Reduce material in- } \\
\text { flow uniformly on } \\
\text { non-critical activi- } \\
\text { ties. }\end{array}$ \\
\hline $\begin{array}{l}\text { Hybrid Strategy } \\
2\end{array}$ & $\begin{array}{l}\text { Recruit workers if they } \\
\text { call in sick; }\end{array}$ & $\begin{array}{l}\text { Always order materials } \\
\text { ahead on critical activity } \\
\text { and order materials on the } \\
\text { most delayed non critical } \\
\text { activity if possible. }\end{array}$ & $\begin{array}{l}\text { Crash the critical activ- } \\
\text { ities and non critical ac- } \\
\text { tivities by allocating ex- } \\
\text { tra labor and material to } \\
\text { activities in case of de- } \\
\text { lay. }\end{array}$ & $\begin{array}{l}\text { Reduce material in- } \\
\text { flow on one non- } \\
\text { critical activity. }\end{array}$ \\
\hline
\end{tabular}

Table 2: Hybrid strategy description

\subsection{Hybrid Strategy}

In summary, the major findings from preliminary and cluster analysis for this particular project include:

- ordering the materials ahead could help mitigate the risks of material delivery failure, reducing the projected cost and duration. It is also especially efficient in minimizing the deviations of projected cost and duration;

- increasing labor and resource input on the critical activity reduces duration;

- whether a strategy is cost effective depends on how the resources are allocated on non-critical activities;

Those findings were context sensitive but provided valuable information to generate better project specific strategies. Users who use the method are able to define the strategies and project context accordingly. New hybrid strategies could be generated by combining the findings. In this study, we were expecting that the newly optimized and generated hybrid strategies were cost and duration efficient while minimizing the deviations. This was because cost and duration management are two important concerns in construction project management. Two hybrid strategies were generated for illustration purposes in this case. In Figures 5 and 6, it was found that the Crash Strategy and the CatchUp Strategy had better performances in managing cost and duration than other strategies. It was a good departure to use the Crash Strategy and the CatchUp Strategy as the base and combine the findings with them to generate new hybrid strategies. The new hybrid strategies were expected to inherit the advantages of crash strategy and catch up strategy and also incorporate the properties from new findings. Actually, the number and the way how new strategies are created are decided by users. They could be generated directly using the findings from data analysis. Combining the data analysis with findings is a shortcut but not necessary.

Hybrid Strategy 1 was based on the Crash Strategy and generated by combining the guidelines in crash Strategy and the data analysis findings. Similarly, Hybrid Strategy 2 was based on the CatchUp Strategy and generated by combining the guidelines in the CatchUp Strategy and the data analysis findings. The disciplines of the new hybrid strategies were defined in Table 2. The material order policy for both Hybrid Strategy 1 and Hybrid Strategy 2 have been altered by incorporating the findings. Material was not allowed to be ordered ahead in original strategies. For resource allocation, the Hybrid Strategy 2 has been altered by emphasizing material order for the most delayed non-critical activities and reducing material flow on only one non critical activity in case of space conflict.

\section{REIMPLEMENTATION OF NEWLY GENERATED HYBRID STRATEGY}

The two newly generated hybrid strategies were expected to perform better than the Crash Strategy and the CatchUp Strategy. For the purpose of validating the method, two newly generated strategies were implemented on ICDMA within the same scenario in the same way the five candidate strategies had been carried out. The performances of all the strategies were summarized and evaluated by cost and duration exceedance and the corresponding deviations.

Figures 7 and 8 illustrated different strategies' performance in managing cost and duration. The performance of the CatchUp Strategy and the Crash Strategy was used as baseline for comparison because two newly generated hybrid strategies were based on them. The newly generated Hybrid 


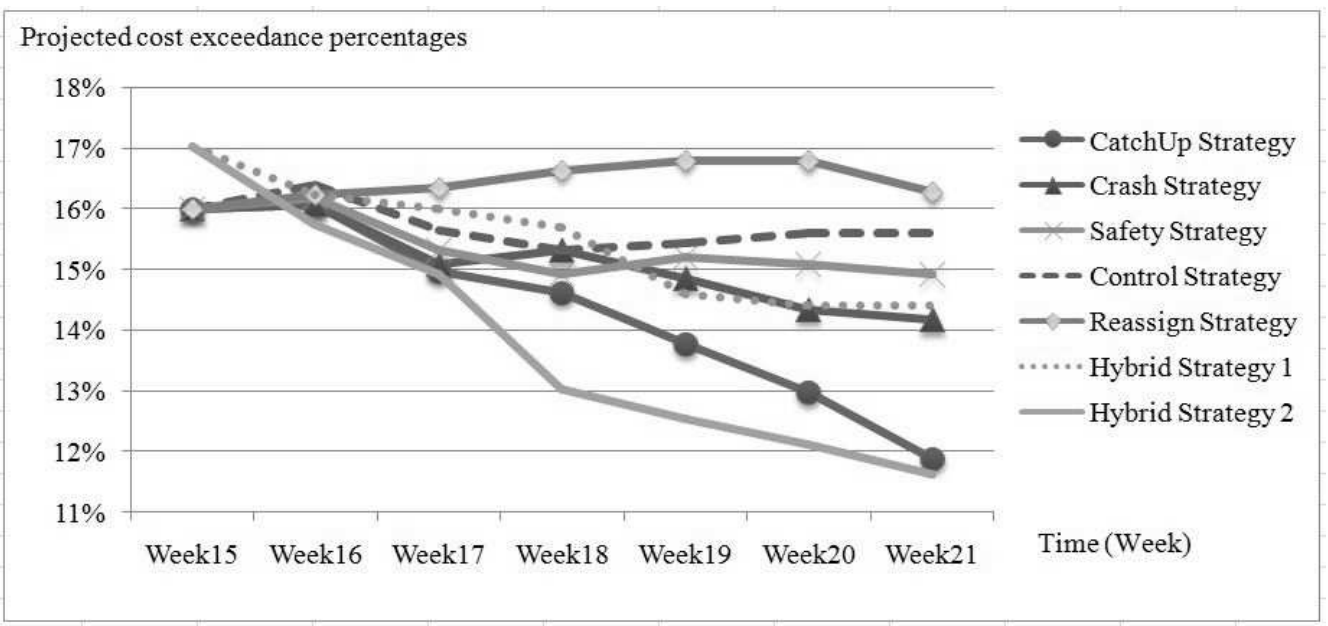

Figure 7: Management of cost exceedance under seven strategies

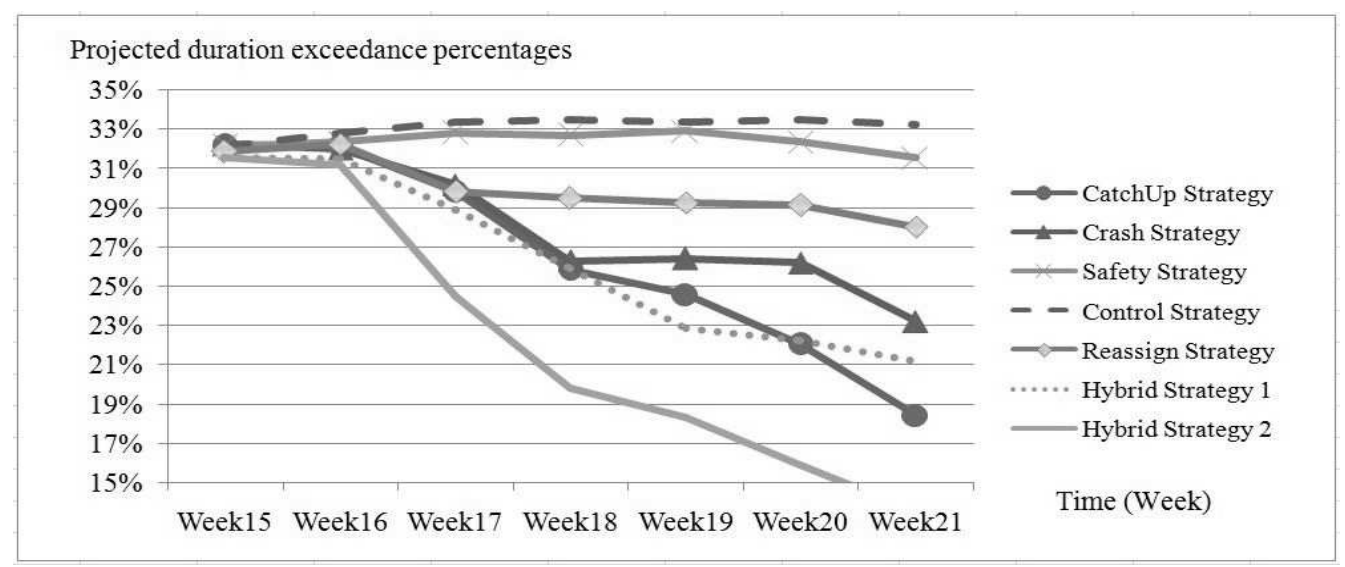

Figure 8: Management of duration exceedance under seven strategies

Strategy 1 had a similar performance to the Crash Strategy in cost control and had a similar performance to the CatchUp Strategy in duration control. Hybrid Strategy 2 had a slightly better performance in cost control and much better performance in duration control than all five candidate strategies and Hybrid Strategy 1. Figures 9 and 10 showed the deviation of predicted cost and duration exceedance percentages under various strategies. Both Hybrid Strategies 1 and 2 had smaller deviations of projected cost exceedance percentages than the CatchUp Strategy and the Crash Strategy. Hybrid Strategy 1 had a smaller deviation of projected duration exceedance percentages than the CatchUp Strategy and the Crash Strategy while Hybrid Strategy 2 had slight advantages over the CatchUp Strategy on deviations of projected duration exceedance percentages.

Compared with the Crash Strategy and the CatchUp Strategy, improved performance was found on the newly generated Hybrid Strategy 1 and 2 in controlling cost and duration as well as minimizing deviations. The differences of those two strategies could be examined to further generate new strategies. Through this iterative optimization process, construction managers are able to optimize and generate strategies for the specific projects before the projects are actually carried out.

\section{DISCUSSION}

A construction project's final outcome is dependent on the sets of day to day decisions occurring in the complete construction process. To study and improve the abilities to make better decisions, it is more critical to investigate the long term interactions of decisions rather than single decision 


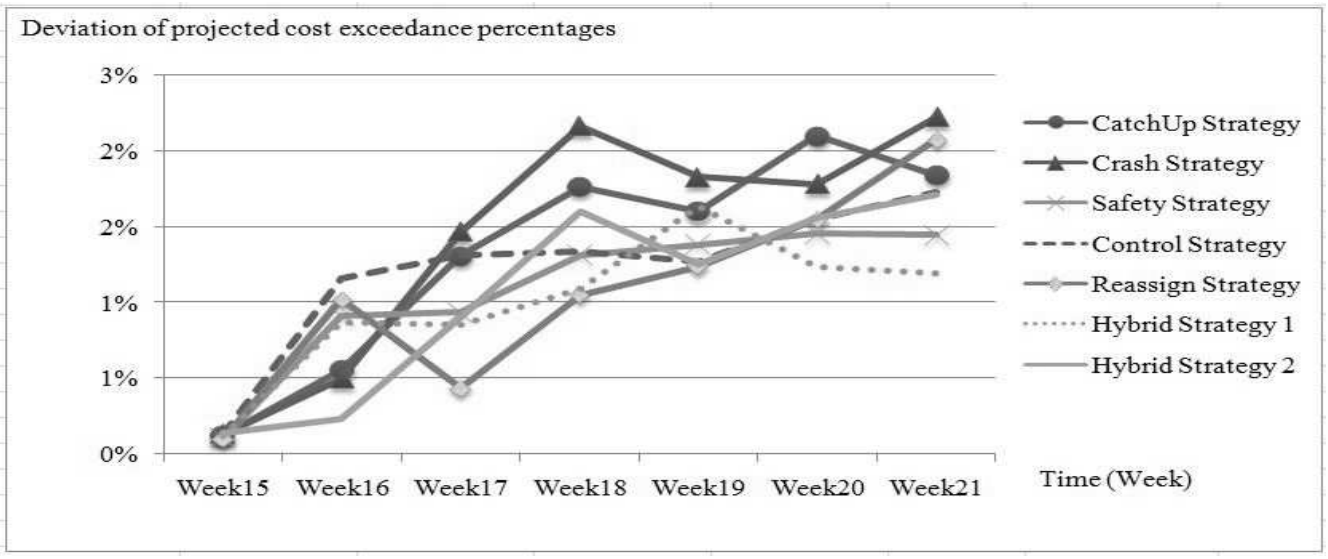

Figure 9: Deviation of projected cost exceedance percentage under seven strategies

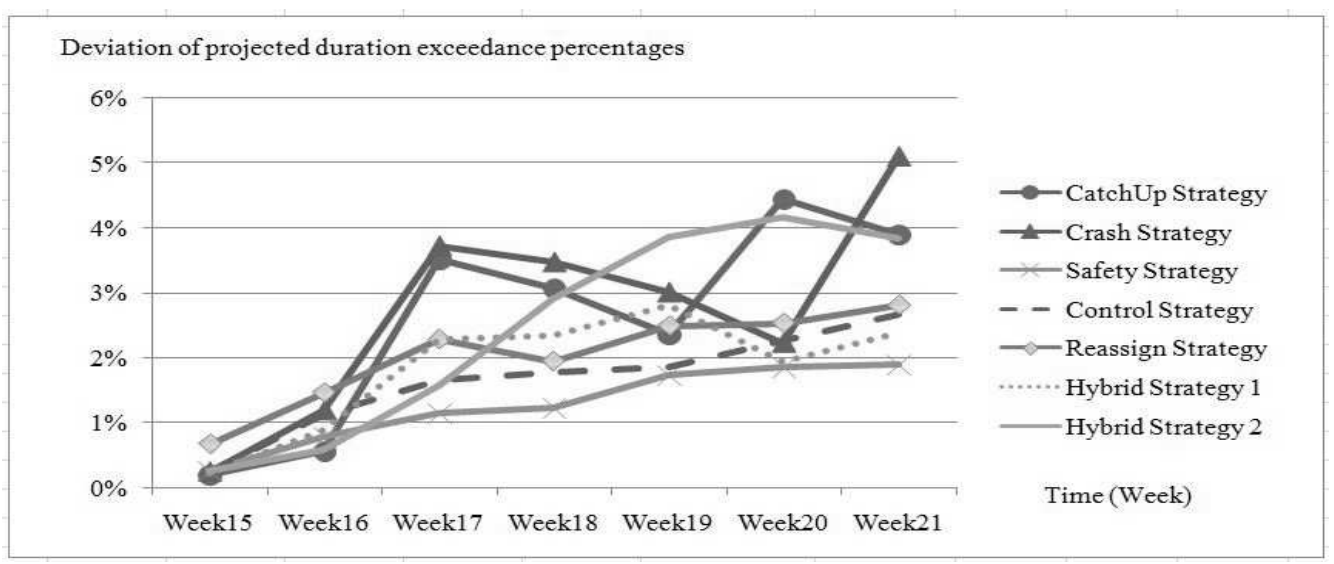

Figure 10: Deviation of projected duration exceedance percentage under seven strategies

output. Decision strategy, a compact representation of day to day decisions and defined as a guideline and direction towards achieving a project outcome, has been investigated as an alternative. Five candidate strategies were implemented in a delayed and cost overrun project and data in the simulation process were collected. The data analysis results showed in this case: (1)ordering the materials ahead could help mitigate the risks of material delivery failure, reducing the projected cost and duration. It was also especially efficient in minimizing the deviations of projected cost and duration; (2)increasing labor and resource input on the critical activity reduced duration; (3)whether a strategy was cost effective depended on how the resources were allocated on non-critical activities. Two hybrid optimized strategies were generated by combining the data analysis results with base strategies. The implementation results showed improvement on the generated strategies. The significance of this research has been proposing a feasible iterative process to optimize and generate strategies through interactive simulation in construction project management. In the future work, stakeholders in construction project will be involved to implement the method and on site data will be collected and analyzed for validation.

\section{ACKNOWLEDGEMENTS}

This work was supported by the NSF grant SES 0624118 and its REU supplement to Amlan Mukherjee. Any opinions, findings and conclusions or recommendations expressed in this material are those of the authors and do not necessarily reflect views of the National Science Foundation. 


\section{REFERENCES}

Anderson, G. R., A. Mukherjee, and N. Onder. 2009. Traversing and querying constraint driven temporal networks to estimate construction contingencies. Automation in Construction 18 (6): 798-813.

Cunningham, A. B., and J. R.. 1984. Water management using interactive simulation. J. Water Resour. Plng. and Mgmt. 110 (3): 310-322.

Cunningham, A. B., and J. R.. 1986. Interactive simulation of water resource systems. J. Water Resour. Plng. and Mgmt. 112 (3): 326-338.

Daccarett, V., and T. Mrozowski. 1999. Aisc digital library: Construction management of steel construction. Available via http://www.aisc.org/content.aspx?id=21250 [accessed 10/26/2009].

Ghodsypour, S., and C. O’Brien. 1998, SEP 20. A decision support system for supplier selection using an integrated analytic hierarchy process and linear programming. International Journal of Production Economics 56-7:199-212.

Herrera-Viedma, E., L. Martinez, F. Mata, and F. Chiclana. 2005, OCT. A consensus support system model for group decision-making problems with multigranular linguistic preference relations. IEEE Transactions on Fuzzy Systems 13 (5): 644-658.

Kaklauskas, A., E. K. Zavadskas, and V. Trinkunas. 2007, MAR. A multiple criteria decision support on-line system for construction. Engineering Applications of Artificial Intelligence 20 (2): 163175.

Kim, J. K., and S. H. Choi. 2001. A utility range-based interactive group support system for multiattribute decision making. Computers \& Operations Research 28 (5): 485 - 503.

Makropoulos, C., D. Butler, and C. Maksimovic. 2003, JAN-FEB. Fuzzy logic spatial decision support system for urban water management. Journal of Water Resources Planning and ManagementASCE 129 (1): 69-77.

Malakooti, B. 1988, mar/apr. A decision support system and a heuristic interactive approach for solving discrete multiple criteria problems. Systems, Man and Cybernetics, IEEE Transactions on 18 (2): $273-284$.

Rojas, E., and A. Mukherjee. 2006. A multi-agent framework for general purpose situational simulations in the construction management domain. Journal of Computing in Civil Engineering 20 (6): 1-12.

Watkins, M., A. Mukherjee, and N. Onder. 2008, December. Using situational simulations to collect and analyze dynamic construction management decision-making data. In WSC '08: Proceedings of the 40th Conference on Winter Simulation, 2377-2386: Winter Simulation Conference, Miami, Florida.

Yates, J. 1993, JUN. Construction decision-support system for delay analysis. Journal of Construction Engineering and management 119 (2): 226-244.

\section{AUTHOR BIOGRAPHIES}

PEI TANG is a Ph.D student at the Department of Civil and Environmental Engineering at Michigan Technological University. His research interests include risk analysis, decision making, artificial intelligence and modeling. His web page can be found via $<$ ht $t \mathrm{p}: / /$ www . cee. mtu.edu $>$.

AMLAN MUKHERJEE is an Assistant Professor at the Department of Civil and Environmental Engineering at Michigan Technological University. His research interests are artificial intelligence technologies such as agent based modeling and temporal logic, construction engineering and management, interactive simulations, construction engineering education, and cognitive modeling of expert decision making. His web page can be found via <http: / / www . cee. mtu. edu>.

NILUFER ONDER is an Associate Professor at the Department of Computer Science at Michigan Technological University. Her research interests are artificial intelligence, planning, planning under uncertainty, and decision making under uncertainty. Her web page can be found via <http: //www. cs.mtu.edu>. 